\title{
Adaptive Coordinated Reception for Multicell MIMO Uplink
}

\author{
Xiaojia Lu, Wei Li, Antti Tölli, and Markku Juntti \\ Centre for Wireless Communications, University of Oulu, P.O. Box 4500, 90014 Oulu, Finland \\ Correspondence should be addressed to Xiaojia Lu, xiaojia.lu@ee.oulu.fi
}

Received 30 June 2010; Revised 9 October 2010; Accepted 16 January 2011

Academic Editor: Francesco Verde

Copyright ( 2011 Xiaojia Lu et al. This is an open access article distributed under the Creative Commons Attribution License, which permits unrestricted use, distribution, and reproduction in any medium, provided the original work is properly cited.

\begin{abstract}
We study the coordinated reception amongst multiple base stations (BS) in the uplink cellular system, where each mobile station (MS) is served by an associated multiple BS set (MBS). Under the individual signal-to-interference-plus-noise ratio (SINR) constraint per MS, power control and receive beamforming are jointly optimized with adaptive MBS selection to minimize the total transmit power. An iterative optimization algorithm is presented accordingly. The joint optimization problem is nonconvex in general, but it can be optimally solved by the proposed algorithm as long as it is feasible. To find the optimum, the proposed algorithm requires the exhaustive search over all BSs per MS. To reduce the complexity in the large-scale cellular network, we propose simplified schemes where a subset of BSs is preselected based on the large-scale fading factors. By limiting the search in the subset, the complexity is reduced significantly. Although the obtained power vector with the simplified algorithm is not optimal, a performance close to the macronetwork full coordination can be achieved by carefully choosing the sizes of the pre-selected BS subset and MBS. The significant advantage is proven by simulations.
\end{abstract}

\section{Introduction}

Uplink communication in the interference limited cellular systems is challenging and has been studied for decades. In the uplink transmission, a mobile station (MS) is not only constrained by its own available resources, but also bears the cochannel interferences (CCI) from other interfering MSs. Transmit power control (TPC) is a conventional technique to achieve a desired system performance. For the uplink of code division multiple access (CDMA) systems, TPC was studied in [1-3], which was utilized to combat the nearfar problem and satisfy a required carrier-to-interference ratio. Under a fixed MS-to-BS assignment, several centralized or decentralized algorithms were proposed. Given a certain signal-to-interference-plus-noise ratio (SINR) requirement per MS, Yates and Huang reformulated uplink TPC as a minimum transmission power (MTP) problem [4]. In their proposal, the BS assignment was considered as another degree of freedom to achieve higher transmit power efficiency. An iterative optimization algorithm was invented therein, which was proven to be able to optimally solve the problem as long as the problem is feasible. This work was extended to three implementation models in [5]. With the multiple antenna extension at BSs, the MTP problem was restudied in [6], where both fixed and adaptive BS assignments were considered. An interesting result is that the joint optimization over TPC and the receive beamforming with either fixed or adaptive assignment can still be optimally solved by using the similar iterative approaches as those in [4], provided that it is a feasible problem. Joint TPC and transmit beamforming problem aiming at the downlink transmissions was studied in [7-9], where the downlinkuplink SINR duality and the coordinated transmit beamformer design based on a semidefinite programming were considered. Using the downlink-uplink duality, it was shown that the same SINR can be simultaneously achieved by the downlink and uplink channels, thereby the joint PC and BS assignment problem in the downlink can also be efficiently solved by the similar approach [10-12].

Multiple-input-multiple-output (MIMO) communications have been studied extensively due to increased spectral efficiency gains. More recently, the cooperative multiBS processing has been shown to achieve further spectral efficiency gains in particular for the cell edge users, 
see, for example, [13-15]. A central controller was set up to collect all information from its connected BSs. By jointly processing the received signals from different cells, more spatial diversity and larger received signal strength can be obtained and meanwhile less CCI. As all connected BSs work in fact as one distributed MIMO system, the set-up is also called coordinated multipoint processing (CoMP) in 3GPP longterm evolution (LTE). The term network MIMO processing is also used. Theoretically, the more BSs join the cooperation in a network MIMO system, the better the CCI mitigation capability is. However, it is difficult to perform a largescale cooperation due to practical limitations. In [16-18], the impact of the limited backhaul capacity has been investigated, where the network MIMO links have a finite capacity but lossless backhaul. Those results were presented based on the information theoretic analysis. Papers in [19-21] studied the impact of the imperfect channel state information (CSI) acquisition. The signaling overhead for acquiring CSI information was taken into account and the results showed that it was the main factor limiting the performance of the network MIMO system rather than the capacity-limited backhaul. Other related literature addressed the crucial problem of the asynchronously arriving signals from multiple transmitters $[22,23]$. All those works suggest that for a large-scale network MIMO system, the only feasible solution is doing partial or local cooperation across a small number of BSs.

In the latest 3GPP LTE Release 10 (LTE-A) standardization process, two local cooperative schemes, that is, the intrasite and inter-site CoMP, have been introduced. The joint processing of the inter-site and intra-site CoMP is within three sectors belonging to the same BS and the different BSs, respectively. The former one requires only physical layer cooperation while the latter one requires crosslayer cooperation. For the inter-site CoMP, fiber connections amongst BSs are needed for the massive upper layer signaling exchange. In both two schemes, the fixed CoMP based on geographic information was considered.

In this paper, the joint reception problem among subset of all BSs combinations is studied. Particularly, we are concerned for the performance gap between the fully cooperative and the partially cooperative network MIMO systems. To fill the gap, we propose an adaptive cooperation scheme instead of the fixed one. In our formulated problem, TPC and receive beamforming are jointly optimized with the associated multi-BS set (MBS) per MS. The diversity reception model introduced in [5] can be regarded as one of the simplest examples of our model, where each BS has only one receive antenna. With adaptive MBS selection per MS, a MTP problem is formulated and an iterative optimization algorithm is present to solve the problem accordingly. The MTP problem is nonconvex, but it can still be optimally solved by the proposed algorithm.

The remainder of this paper is organized as follows. In Section 2, the system model and the problem formulation for minimum power design with adaptive MBS selection are introduced. The algorithm derivation is presented with the convergence and complexity analysis in Section 3. The simulation results are given in Section 4 and the conclusion in Section 5.

\section{System Model and Problem Formulation}

2.1. The Multicell Multiuser System. The considered cellular system consists of $K$ MSs and B BSs in flat fading channels. Assuming that all MSs have one transmit antenna while BS $i$ has $N_{i}$ receive antennas, the received signal $\mathbf{r}_{i}$ at the $i$ th BS is written as

$$
\mathbf{r}_{i}=\sum_{k=1}^{K} \sqrt{p_{k}} \mathbf{h}_{k, i} s_{k}+\mathbf{n}_{i}
$$

where $p_{k}$ is the transmit power of MS $k, \mathbf{h}_{k, i} \in \mathbb{C}^{N_{i} \times 1}$ is the complex channel response from the $k$ th MS to the $i$ th BS, $s_{k}$ is the transmitted symbol of MS $k$ with average power normalized to 1 , and $\mathbf{n}_{i}$ is the additive circular symmetric white Gaussian noise (AWGN) vector at BS $i$ with distribution $\mathcal{C} \mathcal{N}\left(0, \sigma_{i}^{2}\right)$ on each receive antenna.

Assuming that MS $k$ is simultaneously served by a set of chosen BSs, a set of linear beamformers can be utilized to extract its signal from those BSs, that is,

$$
\begin{aligned}
\hat{\boldsymbol{s}}_{k}= & \sum_{i \in \pi_{k}} \mathbf{w}_{k, i}^{\mathrm{H}} \mathbf{r}_{i} \\
= & \sum_{i \in \pi_{k}} \sqrt{p_{k}} \mathbf{w}_{k, i}^{\mathrm{H}} \mathbf{h}_{k, i} s_{k} \\
& +\sum_{i \in \pi_{k}}\left(\sum_{k^{\prime}=1, k^{\prime} \neq k}^{K} \sqrt{p_{k^{\prime}}} \mathbf{w}_{k, i}^{\mathrm{H}} \mathbf{h}_{k^{\prime}, i} s_{k^{\prime}}+\mathbf{w}_{k, i}^{\mathrm{H}} \mathbf{n}_{i}\right),
\end{aligned}
$$

where $\mathbf{w}_{k, i} \in \mathbb{C}^{N_{i} \times 1}$ is the receive beamforming vector for MS $k$ 's received signal at BS $i,(\cdot)^{\mathrm{H}}$ denotes the Hermitian operator and $\pi_{k}$ is the MBS serving MS $k$. The elements in $\pi_{k}=\left\{\pi_{k}(1), \pi_{k}(2), \ldots, \pi_{k}\left(\left|\pi_{k}\right|\right)\right\}$ are the indices of the BSs jointly providing service to MS $k$, where $\left|\pi_{k}\right|$ denotes cardinality of $\pi_{k}$.

The interference-plus-noise power that MS $k$ experiences can be calculated as

$$
\mathbf{I}_{k}\left(\mathbf{p}, \pi_{k}, \mathbf{w}_{k}\right)=\sum_{k^{\prime}=1, k^{\prime} \neq k}^{K}\left|\sum_{i \in \pi_{k}} \mathbf{w}_{k, i}^{\mathrm{H}} \mathbf{h}_{k^{\prime}, i}\right|^{2} p_{k^{\prime}}+\sum_{i \in \pi_{k}}\left\|\mathbf{w}_{k, i}\right\|^{2} \sigma_{i}^{2},
$$

where $|\cdot|$ denotes the absolute value and $\|\cdot\|$ the standard Euclidean norm. Let $\mathbf{p}=\left[\begin{array}{llll}p_{1} & p_{2} & \cdots & p_{K}\end{array}\right]^{T}$ be the stacked vector including all MSs transmit powers; $\mathbf{w}_{k}$ is the stacked beamformer of MS $k$, that is, $\left[\mathbf{w}_{k, \pi_{k}(1)}^{T} \mathbf{w}_{k, \pi_{k}(2)}^{T} \cdots \mathbf{w}_{k, \pi_{k}\left(\left|\pi_{k}\right|\right)}^{T}\right]^{T}$.

2.2. Problem Formulation. Using (3), the effective SINR of MS $k$ can be written as

$$
\Gamma_{k, \pi_{k}, \mathbf{w}_{k}}=\frac{\left|\sum_{i \in \pi_{k}} \mathbf{w}_{k, i}^{\mathrm{H}} \mathbf{h}_{k, i}\right|^{2} p_{k}}{\mathbf{I}_{k}\left(\mathbf{p}, \pi_{k}, \mathbf{w}_{k}\right)}
$$


The considered MTP problem becomes

$$
\begin{array}{cl}
\underset{\mathbf{w}_{k, i}, \pi_{k}, p_{k}}{\operatorname{minimize}} & P_{\text {tot }}=\sum_{k=1}^{K} p_{k} \\
\text { subject to } & \Gamma_{k, \pi_{k}, \mathbf{w}_{k}} \geq \gamma_{k}, \\
& p_{k} \leq P_{k},
\end{array}
$$

where $\gamma_{k}$ is the minimum SINR requirement of MS $k$ and $P_{k}$ MS $k$ 's maximum transmit power. The optimization problem (5) is a nonconvex problem in general. When $\left|\pi_{k}\right|=1$, it becomes exactly the same as the one in [6]. On the other hand, when all $\left|\pi_{k}\right|=B$, it is reduced to coherent beamforming across all BSs [5].

By defining a mapping function $m(\mathbf{p})=\left[m_{1}(\mathbf{p}) \quad m_{2}(\mathbf{p})\right.$ $\left.\cdots m_{K}(\mathbf{p})\right]^{T}$, where

$$
m_{k}(\mathbf{p})=\min _{\pi_{k}, \mathbf{w}_{k}} \frac{\mathbf{I}_{k}\left(\mathbf{p}, \pi_{k}, \mathbf{w}_{k}\right)}{\left|\sum_{i \in \pi_{k}} \mathbf{w}_{k, i}^{\mathrm{H}} \mathbf{h}_{k, i}\right|^{2}} \gamma_{k},
$$

we have the following result.

Lemma 1. $m(\mathbf{p})$ satisfies three properties of a standard interference function.

(1) Positivity: $m(\mathbf{p})>\mathbf{0}$, where $\succ$ denotes element-wise larger.

(2) Monotonicity: if $\mathbf{p} \succeq \mathbf{p}^{\prime}$, then $m(\mathbf{p}) \succeq m\left(\mathbf{p}^{\prime}\right)$, where $\succeq$ is element-wise no smaller.

(3) Scalability: for any $\alpha>1, \alpha m(\mathbf{p}) \succ m(\alpha \mathbf{p})$.

Proof. (1) Positivity: the term $\sum_{k^{\prime}=1, k^{\prime} \neq k}^{K}\left|\sum_{i \in \pi_{k}} \mathbf{w}_{k, i}^{\mathrm{H}} \mathbf{h}_{k^{\prime}, i}\right|^{2}$ $p_{k^{\prime}} \geq 0$ and $\left\|\mathbf{w}_{k, i}\right\|^{2} \sigma_{i}^{2}>0$ due to the fact that the noise power and link gain can not be zero. Thus, $m_{k}(\mathbf{p})$ is always positive.

(2) Monotonicity: given two power vectors $\mathbf{p}$ and $\mathbf{p}^{\prime}$ with $\mathbf{p} \succeq \mathbf{p}^{\prime}$, it is evident that for any $\pi_{k}$ and $\mathbf{w}_{k}$

$$
\mathbf{I}_{k}\left(\mathbf{p}, \pi_{k}, \mathbf{w}_{k}\right) \geq \mathbf{I}_{k}\left(\mathbf{p}^{\prime}, \pi_{k}, \mathbf{w}_{k}\right) .
$$

Since the term $\left|\sum_{i \in \pi_{k}} \mathbf{w}_{k, i}^{\mathrm{H}} \mathbf{h}_{k, i}\right|^{2}$ is the same, we have

$$
\begin{aligned}
m_{k}(\mathbf{p}) & =\min _{\pi_{k}, \mathbf{w}_{k}} \frac{\mathbf{I}_{k}\left(\mathbf{p}, \pi_{k}, \mathbf{w}_{k}\right)}{\left|\sum_{i \in \pi_{k}} \mathbf{w}_{k, i}^{\mathrm{H}} \mathbf{h}_{k, i}\right|^{2}} \\
& \geq \min _{\pi_{k}, \mathbf{w}_{k}} \frac{\mathbf{I}_{k}\left(\mathbf{p}^{\prime}, \pi_{k}, \mathbf{w}_{k}\right)}{\left|\sum_{i \in \pi_{k}} \mathbf{w}_{k, i}^{\mathrm{H}} \mathbf{h}_{k, i}\right|^{2}} \\
& =m_{k}\left(\mathbf{p}^{\prime}\right) .
\end{aligned}
$$

The monotonicity holds.

(3) Scalability: for any $\alpha>1$, we have

$$
\begin{aligned}
& \alpha m_{k}(\mathbf{p}) \\
& =\alpha \min _{\pi_{k}, \mathbf{w}_{k}} \frac{\mathbf{I}_{k}\left(\mathbf{p}, \pi_{k}, \mathbf{w}_{k}\right)}{\left|\sum_{i \in \pi_{k}} \mathbf{w}_{k, i}^{\mathrm{H}} \mathbf{h}_{k, i}\right|^{2}} \gamma_{k}=\min _{\pi_{k}, \mathbf{w}_{k}} \frac{\alpha \mathbf{I}_{k}\left(\mathbf{p}, \pi_{k}, \mathbf{w}_{k}\right)}{\left|\sum_{i \in \pi_{k}} \mathbf{w}_{k, i}^{\mathrm{H}} \mathbf{h}_{k, i}\right|^{2}} \gamma_{k} \\
& =\min _{\pi_{k}, \mathbf{w}_{k}} \frac{\alpha \sum_{k^{\prime} \neq k}\left|\sum_{i \in \pi_{k}} \mathbf{w}_{k, i}^{\mathrm{H}} \mathbf{h}_{k^{\prime}, i}\right|^{2} p_{k^{\prime}}+\left.\alpha \sum_{i \in \pi_{k}}|| \mathbf{w}_{k, i}\right|^{2} \sigma_{i}^{2}}{\left|\sum_{i \in \pi_{k}} \mathbf{w}_{k, i}^{\mathrm{H}} \mathbf{h}_{k, i}\right|^{2}} \gamma_{k} \\
& >\min _{\pi_{k}, \mathbf{w}_{k}} \frac{\sum_{k^{\prime} \neq k}\left|\sum_{i \in \pi_{k}} \mathbf{w}_{k, i}^{\mathrm{H}} \mathbf{h}_{k^{\prime}, i}\right|^{2} \alpha p_{k^{\prime}}+\left.\sum_{i \in \pi_{k}}|| \mathbf{w}_{k, i}\right|^{2} \sigma_{i}^{2}}{\left|\sum_{i \in \pi_{k}} \mathbf{w}_{k, i}^{\mathrm{H}} \mathbf{h}_{k, i}\right|^{2}} \gamma_{k},
\end{aligned}
$$

where the inequality comes from the fact that the term $\left|\sum_{i \in \pi_{k}} \mathbf{w}_{k, i}^{\mathrm{H}} \mathbf{h}_{k, i}\right|^{2}$ is positive and $\alpha>1$.

As the proof holds for all $k$, our proof completes.

\section{Minimum Power Beamforming with Adaptive MBS Selection}

3.1. Algorithm Derivation. The minimum transmit power of an MS, jointly optimized over beamforming and MBS in (6), is shown to be a standard interference function. This allows us to follow the standard power control approach [4]. The MTP problem with adaptive MBS selection can be also solved by using the iterative optimization algorithm, where at each iteration the power vector is updated by

$$
\mathbf{p}^{[t+1]}=m\left(\mathbf{p}^{[t]}\right) .
$$

Recall that $m\left(\mathbf{p}^{[t]}\right)$, which can be calculated from (6), is the minimum power vector that is optimized over MBS and beamforming space to meet the SINR target. The optimal receive beamforming matrix for multiuser joint power control and BS assignment is known to be minimummean square error filter [24], which is given by

$$
\begin{aligned}
& {\left[\begin{array}{c}
\mathbf{w}_{k, \pi_{k}(1)}^{[t]} \\
\vdots \\
\mathbf{w}_{k, \pi_{k}\left(\left|\pi_{k}\right|\right)}^{[t]}
\end{array}\right]} \\
& =\arg \max _{\mathbf{w}_{k, i}} \frac{\left|\sum_{i \in \pi_{k}} \mathbf{w}_{k, i}^{\mathrm{H}} \mathbf{h}_{k, i}\right|^{2} \widetilde{p}_{k}^{[t-1]}}{\sum_{k^{\prime} \neq k}\left|\sum_{i \in \pi_{k}} \mathbf{w}_{k, i}^{\mathrm{H}} \mathbf{h}_{k^{\prime}, i}\right|^{2} \tilde{p}_{k^{\prime}}^{[t-1]}+\left.\sum_{i \in \pi_{k}}|| \mathbf{w}_{k, i}\right|^{2} \sigma_{i}^{2}} \\
& =\left(\sum_{k^{\prime} \neq k} p_{k^{\prime}}^{[t-1]}\left[\begin{array}{c}
\mathbf{h}_{k^{\prime}, \pi_{k}(1)} \\
\vdots \\
\mathbf{h}_{k^{\prime}, \pi_{k}\left(\left|\pi_{k}\right|\right)}
\end{array}\right]\left[\mathbf{h}_{k^{\prime}, \pi_{k}(1)}^{\mathrm{H}} \cdots \mathbf{h}_{k^{\prime}, \pi_{k}\left(\left|\pi_{k}\right|\right)}^{\mathrm{H}}\right]\right. \\
& \left.+\left[\begin{array}{ccc}
\sigma_{\pi_{k}(1)}^{2} \mathbf{I} & & \\
& \ddots & \\
& & \sigma_{\pi_{k}\left(\left|\pi_{k}\right|\right)}^{2} \mathbf{I}
\end{array}\right]\right)^{-1}\left[\begin{array}{c}
\mathbf{h}_{k, \pi_{k}(1)} \\
\vdots \\
\mathbf{h}_{k, \pi_{k}\left(\left|\pi_{k}\right|\right)}
\end{array}\right] .
\end{aligned}
$$

Substituting $m\left(\mathbf{p}^{[t]}\right)$ by (6) and $\mathbf{w}_{k}$ by (11), a detailed description of the iterative joint optimization algorithm is given as Algorithm 1.

3.2. Optimality and Convergence Analysis in Feasible Case. We have formulated the optimization problem in (5) and give a power control approach in Section 3.1. In this subsection, we are going to study the existence and convergence of the global optimal solution. First, we give the following theorem.

Theorem 1. If (5) is feasible, then the minimum power vector $\mathbf{p}^{\star}$ must be a fixed point of the mapping function $m(\mathbf{p})$ and the fixed point is unique. 
Input: $\mathbf{h}_{k}, P_{k}, \sigma_{i}^{2}$ and $\gamma_{k}$.

(1) Initialization: Set the iteration index $t=0$. Set $\tilde{p}_{k}^{[0]}=0$ for all $k$.

(2) Set $t=t+1$, for each MS $k$

(a) Calculate $\left\{\mathbf{w}_{k, \pi_{k}(1)}, \mathbf{w}_{k, \pi_{k}(2)}, \ldots, \mathbf{w}_{k, \pi_{k}\left(\left|\pi_{k}\right|\right)}\right\}$ for all possible MBS sets $\pi_{k}$ by using (11).

(b) Given the beamforming vectors for each possible $\pi_{k}$, compute the minimum required transmit power $p_{k, \pi_{k}}$ to fulfill the SINR constraint $\gamma_{k}$ as

(c) Select $\tilde{\pi}_{k}^{[t]}$ that requires the least transmit power

$$
p_{k, \pi_{k}}=\frac{\sum_{k^{\prime} \neq k}\left|\sum_{i \in \pi_{k}} \mathbf{w}_{k, i}^{\mathrm{H}} \mathbf{h}_{k^{\prime}, i}\right|^{2} \tilde{p}_{k^{\prime}}^{[t-1]}+\sum_{i \in \pi_{k}}\left\|\mathbf{w}_{k, i}\right\|^{2} \sigma_{i}^{2}}{\left|\sum_{i \in \pi_{k}} \mathbf{w}_{k, i}^{\mathrm{H}} \mathbf{h}_{k, i}\right|^{2}} \gamma_{k}
$$

$$
\tilde{\pi}_{k}^{[t]}=\arg \min _{\pi_{k}} p_{k, \pi_{k}}
$$

(d) Update $\left\{\tilde{\mathbf{w}}_{k, \tilde{\pi}_{k}^{[t]}[1)}^{[t]}, \tilde{\mathbf{w}}_{k, \tilde{\pi}_{k}^{[t]}(2)}^{[t]}, \ldots, \tilde{\mathbf{w}}_{k, \tilde{\pi}_{k}^{[t]}\left(||_{\pi}^{[t]} \mid\right)}^{[t]}\right.$ and $\tilde{p}_{k}^{[t]}$ accordingly, that is,

$$
\begin{aligned}
& \left\{\tilde{\mathbf{w}}_{k, \tilde{\pi}_{k}^{[t]}(1)}^{[t}, \widetilde{\mathbf{w}}_{k, \tilde{\pi}_{k}^{[t]}(2)}^{[t]}, \ldots, \tilde{\mathbf{w}}_{k, \tilde{\pi}_{k}^{[t]}\left(\left|\tilde{\pi}_{k}^{[t]}\right|\right)}\right\} \\
& =\left\{\mathbf{w}_{k, \tilde{\pi}_{k}^{[t]}(1)}, \mathbf{w}_{k, \tilde{\pi}_{k}^{[t]}(2)}, \ldots, \mathbf{w}_{k, \tilde{\pi}_{k}^{[t]}\left(\left|\tilde{\pi}_{k}\right|\right)}\right\}, \\
& \tilde{p}_{k}^{[t]}=p_{k, \tilde{\pi}_{k}^{[t]}} .
\end{aligned}
$$

(e) If any $\tilde{p}_{k}^{[t]}>P_{k}$, stop iterations since the SINR constraints are infeasible.

Output:

(3) If the difference between $\sum_{k=1}^{K} \tilde{p}_{k}^{[t]}$ and $\sum_{k=1}^{K} \tilde{p}_{k}^{[t-1]}$ is less than a threshold $\epsilon$, for example, $5 e-3$, stop and output the result. Otherwise go to step (2).

(1) Infeasible case: an infeasible indicator.

(2) Feasible case: $\tilde{p}_{k}^{[t]},\left\{\tilde{\mathbf{w}}_{k, \tilde{\pi}_{k}^{[t]}(1)}^{[t]}, \tilde{\mathbf{w}}_{k, \tilde{\pi}_{k}^{[t]}(2)}^{[t]}, \ldots, \tilde{\mathbf{w}}_{k,,_{k}^{[t]}\left(\left|\tilde{\pi}_{k}^{[t]}\right|\right)}\right\}$ and $\tilde{\pi}_{k}^{[t]}, 1<k<K$.

Algorithm 1: Joint TPC and receive beamforming with adaptive MBS selection.

Proof. From the minimum SINR constraint of (5), after a minor modification, we have

$$
\mathbf{p}^{\star}=\left[\begin{array}{c}
p_{1}^{\star} \\
p_{2}^{\star} \\
\vdots \\
p_{K}^{\star}
\end{array}\right] \succeq\left[\begin{array}{c}
m_{1}\left(\mathbf{p}^{\star}\right) \\
m_{2}\left(\mathbf{p}^{\star}\right) \\
\vdots \\
m_{K}\left(\mathbf{p}^{\star}\right)
\end{array}\right]=m\left(\mathbf{p}^{\star}\right) .
$$

Given that problem (5) is feasible and $\mathbf{p}^{\star}$ is the minimum power vector, without loss of generality, suppose that the equality does not hold for MS 1, for example, we can always construct another feasible power vector

$$
\mathbf{p}^{\star \star}=\left[\begin{array}{c}
p_{1}^{\star \star} \\
p_{2}^{\star \star} \\
\vdots \\
p_{K}^{\star \star}
\end{array}\right]=\left[\begin{array}{c}
m_{1}\left(\mathbf{p}^{\star}\right) \\
p_{2}^{\star} \\
\vdots \\
p_{K}^{\star}
\end{array}\right] .
$$

It is evident that $p_{1}^{\star \star}=m_{1}\left(\mathbf{p}^{\star}\right)<p_{1}^{\star}$ and $\sum_{k=1}^{K} p_{k}^{\star \star}$ is smaller than $\sum_{k=1}^{K} p_{k}^{\star}$, which conflicts with the assumption that $\mathbf{p}^{\star}$ is the optimum. Therefore, the equality must hold, that is, $\mathbf{p}^{\star}$ is a fixed point of $m(\mathbf{p})$. Next, following the approach in [6], we assume that there are two different fixed points $\mathbf{p}^{\star, 1}$ and $p^{\star, 2}$, which are also positive. Without loss of generality, we also assume that $\mathbf{p}^{\star, 1}$ has at least one element larger than $\mathbf{p}^{\star, 2}$. Thus, we must be able to find an index

$$
l=\underset{k}{\arg \max } \frac{p_{k}^{\star}, 1}{p_{k}^{\star, 2}}
$$

and a scalar $\alpha=p_{l}^{\star, 1} / p_{l}^{\star, 2}>1$. Then, we can construct a new vector $\alpha \mathbf{p}^{\star, 2}$, where $\alpha p_{l}^{\star, 2}=\mathbf{p}_{l}^{\star, 1}$ and $\alpha \mathbf{p}_{k}^{\star, 2} \geq \mathbf{p}_{k}^{\star, 1}$ for $k \neq l$. However, for $\mathbf{p}_{l}^{\star, 1}$, by the scalability and monotonicity properties, we also have

$$
\mathbf{p}_{l}^{\star, 1}=m_{l}\left(\mathbf{p}^{\star, 1}\right)<m_{l}\left(\alpha \mathbf{p}^{\star, 2}\right)<\alpha m_{l}\left(\mathbf{p}^{\star, 2}\right)=\alpha \mathbf{p}_{l}^{\star, 2} .
$$

The last equality comes from the fact that $\mathbf{p}_{l}^{\star, 2}$ is also a fixed point. As this contradicts the fact that $\mathbf{p}_{l}^{\star, 1}=\alpha \mathbf{p}_{l}^{\star, 2}$, thus the fixed point must be unique. The proof is completed.

To prove the convergence, we first define $\mathbf{p}_{\mathbf{p}^{\prime}}^{[t]}$ as the power vector produced by Algorithm 1 at iteration $t$ with the initial power vector $\mathbf{p}^{\prime}$. Then, we have the following theorems.

Theorem 2. If (5) is feasible and the initial power vector $\mathbf{p}^{\prime}$ is a feasible solution, the output power vector of Algorithm 1 must monotonically decrease after every iteration and will converge to the optimal solution $\mathbf{p}^{\star}$.

Proof. First, the SINR constraint determines that $\mathbf{p}_{\mathbf{p}^{\prime}}^{[0]}=\mathbf{p}^{\prime} \succeq$ $m\left(\mathbf{p}^{\prime}\right)=\mathbf{p}_{\mathbf{p}^{\prime}}^{[1]}$ as $\mathbf{p}^{\prime}$ is a feasible solution. By the monotonicity property, we have $\mathbf{p}_{\mathbf{p}^{\prime}}^{[0]} \succeq m\left(\mathbf{p}_{\mathbf{p}^{\prime}}^{[0]}\right)=\mathbf{p}_{\mathbf{p}^{\prime}}^{[1]} \succeq m\left(\mathbf{p}_{\mathbf{p}^{\prime}}^{[1]}\right)=\mathbf{p}_{\mathbf{p}^{\prime}}^{[2]} \succeq$ $\cdots \succeq m\left(\mathbf{p}_{\mathbf{p}^{\prime}}^{[t-1]}\right)=\mathbf{p}_{\mathbf{p}^{\prime}}^{[t]}$. Obviously, $\mathbf{p}_{\mathbf{p}^{\prime}}^{[t]}$ is monotonically decreasing with $t$. By the positivity property, the power vector is lower bounded by zero. Thus, it must converge to a fixed point as the index $t$ increases. Based on the fact that the fixed point is unique, the proof is completed.

Theorem 3. Algorithm 1, with an initial power vector $\mathbf{p}^{\prime}=$ 0 , produces a monotonic increasing power vector sequence. 
Moreover, if (5) is feasible, the sequence must converge to the optimal solution $\mathbf{p}^{\star}$.

Proof. First, $\mathbf{0} \prec m(\mathbf{0})$ must hold because of the positivity property, where $\prec$ denotes the element-wise smaller. Then using the monotonicity property, we have $\mathbf{0}=\mathbf{p}_{0}^{[0]} \preceq$ $m\left(\mathbf{p}_{0}^{[0]}\right)=\mathbf{p}_{0}^{[1]} \preceq m\left(\mathbf{p}_{0}^{[1]}\right)=\mathbf{p}_{0}^{[2]} \preceq \cdots \preceq m\left(\mathbf{p}_{0}^{[t-1]}\right)=$ $\mathbf{p}_{0}^{[t]}$. Obviously, the value of the produced vector sequence is increasing. Assuming that the problem is feasible and $\mathbf{p}^{*}$ is the minimum power vector, we can easily obtain a sequence with Algorithm 1 with $\mathbf{p}^{\prime}=\mathbf{p}^{\star}$, that is, $\mathbf{p}^{\star}=\mathbf{p}_{\mathbf{p}^{\star}}^{[0]}=m\left(\mathbf{p}^{\star}\right)=$ $\mathbf{p}_{\mathbf{p}^{\star}}^{[1]}=m\left(\mathbf{p}^{\star}\right)=\mathbf{p}_{\mathbf{p}^{\star}}^{[2]}=\cdots=m\left(\mathbf{p}^{\star}\right)=\mathbf{p}_{\mathbf{p}^{\star}}^{[t]}$. The equality is from the fact that $\mathbf{p}^{\star}$ is the fixed point. By using the monotonicity property, we have $\mathbf{p}_{\mathbf{p}^{\star}}^{[t]} \succ \mathbf{p}_{0}^{[t]}$. We complete the proof.

Theorem 4. With any initial power vector $\mathbf{p}^{\prime}$, despite $\mathbf{p}^{\prime}$ is feasible or not, the produced power sequence from Algorithm 1 converges to the optimal solution $\mathbf{p}^{\star}$, as long as (5) is feasible.

Proof. First, for any $\mathbf{p}^{\prime}$, we can always find a scalar $\alpha>1$ satisfying $\mathbf{p}^{\prime} \preceq \alpha \mathbf{p}^{\star}$. Using the scalability property, we know that $\alpha \mathbf{p}^{\star}$ must be a feasible solution as well. Since $0 \preceq \mathbf{p}^{\prime} \preceq$ $\alpha \mathbf{p}^{\star}$, we have $\mathbf{p}_{\mathbf{0}}^{[1]} \preceq \mathbf{p}_{\mathbf{p}^{\prime}}^{[1]} \preceq \mathbf{p}_{\alpha \mathbf{p}^{\star}}^{[1]}, \mathbf{p}_{\mathbf{0}}^{[2]} \preceq \mathbf{p}_{\mathbf{p}^{\prime}}^{[2]} \preceq \mathbf{p}_{\alpha \mathbf{p}^{\star}}^{[2]}$ and so on, based on the monotonicity property. According to Theorems 2 and 3 , both $\mathbf{p}_{0}^{[1]}$ and $\mathbf{p}_{\alpha \mathbf{p}^{\star}}^{[2]}$ converge to $\mathbf{p}^{\star}$ as the index $t$ is increased, and the middle part must converge to $\mathbf{p}^{\star}$ as well. Our proof completes.

3.3. Discussion on Convergence. When the problem is feasible, we proved the convergence of Algorithm 1 in Section 3.2. In practical systems, the SINR constraint is not always achievable due to large amount of interference or the time selectivity of the wireless channels. In this case, we have following corollary.

Corollary 1. For any initial power vector $\mathbf{p}^{\prime}$, the power vector sequence produced by Algorithm 1 approaches infinity, that is, $P_{\text {tot }} \rightarrow+\infty$, if (5) is infeasible.

Proof. The proof is straightforward from the previous theorems. First, Theorem 3 points out that the algorithm always generates an increasing sequence when using $\mathbf{p}^{\prime}=\mathbf{0}$. As the problem is not feasible and the produced sequence is not upper bounded, $P_{\text {tot }} \rightarrow+\infty$. Then using the proof of Theorem 4, we know that the produced sequence by Algorithm 1 with arbitrary $\mathbf{p}^{\prime}$ is element-wise no smaller than the one with $\mathbf{p}^{\prime}=\mathbf{0}$. As a result, it must also approach infinity. This completes the proof.

Although Algorithm 1 may not always converge to a fixed solution, it can be terminated by a cutoff threshold, for example, the maximum transmit power constraint in practical systems. If Algorithm 1 is initialized with zero power vector, the value of the generated sequence is always increasing. The value of the updated power vector will either increase to the optimal solution or reach/exceed the cutoff value $P_{k}$. The latter case denotes the infeasibility of the problem and can be easily detected by step 2(e) in Algorithm 1.

3.4. Complexity Discussion and Algorithm Simplification. Given $\left|\pi_{k}\right|$ fixed, Algorithm 1 can optimally solve the problem, but its complexity is still high due to the exhaustive search over all BSs combination set to find the optimal MBS for each MS. At each iteration, the number of candidates in the exhaustive search is given by $K\left(\prod_{l=1}^{B} l / \prod_{m=1}^{\left|\pi_{k}\right|} m \prod_{n=1}^{B-\left|\pi_{k}\right|} n\right)$, which dominates the total computational complexity if the network size $B$ is large. On the other hand, in order to achieve larger spatial diversity gain, larger size of MBS, for example, $\left|\pi_{k}\right|=3$, is required, which also increases the complexity. To achieve a better tradeoff between the performance and the complexity, we propose to use the BS preselection to limit the number of the candidate BSs to be searched per MS. Practically, a central controller can pre-select $R_{k}$ BSs for MS $k$ based on measured received signal strengths (RSSs) at each $\mathrm{BS}$, where $\left|\pi_{k}\right| \leq R_{k} \leq B$. Then, the central controller in the network runs Algorithm 1 but limits the adaptive MBS selection over those $R_{k}$ BSs instead of all BSs. By doing so, the number of iterations of the exhaustive search for MS $k$ reduces to $\sum_{k=1}^{K}\left(\prod_{l=1}^{R_{k}} l / \prod_{m=1}^{\left|\pi_{k}\right|} m \prod_{n=1}^{R_{k}-\left|\pi_{k}\right|} n\right)$ and we are able to flexibly balance the complexity and the performance.

We summarize the optimal and simplified schemes as follows.

Algorithm 1 (full set selection). We set $R_{k}=B$ which means that an exhaustive search over all BSs is carried out to find the optimal $\pi_{k}$ for MS $k$.

Algorithm 2 (single element selection). We set $R_{k}=\left|\pi_{k}\right|$, which means that the selected $\left|\pi_{k}\right|$ BSs are those which provide $\left|\pi_{k}\right|$ largest RSS values for MS $k$. Compared to Algorithms 1 and 2 does not need exhaustive search, and is thereby remarkably simpler.

Algorithm 3 (partial set selection). We consider the case where $\left|\pi_{k}\right|<R_{k}<B$ and $R_{1}=R_{2}=\cdots=R_{K}$. This scheme is significantly computationally more efficient than Algorithm 1 when $R_{k}$ is far smaller than $B$, but it is still more complex than Algorithm 2. However, it will show performance close to that of Algorithm 1 in the simulations.

Algorithm 4 (adaptive-sized partial set selection). In this scheme, $R_{k}$ is also dynamically changed according to the BSs' RSS values. To do so, we set a threshold $P_{L}$ for the BS preselection. Only those BSs whose RSS differences to the strongest BS are less than $P_{L}$ will be taken into account. In this case, the number of exhaustive search of each MS is not fixed and could be different from time to time.

\section{Simulation Results}

The proposed schemes with different sizes of $\pi_{k}$ are evaluated by the system-level simulations. The uplink intra-inter-site CoMPs are also compared with the proposed algorithms. In the simulation, we consider a cellular system containing 19 BSs with 3 sectors each, adding up to 57 sectors in total. 


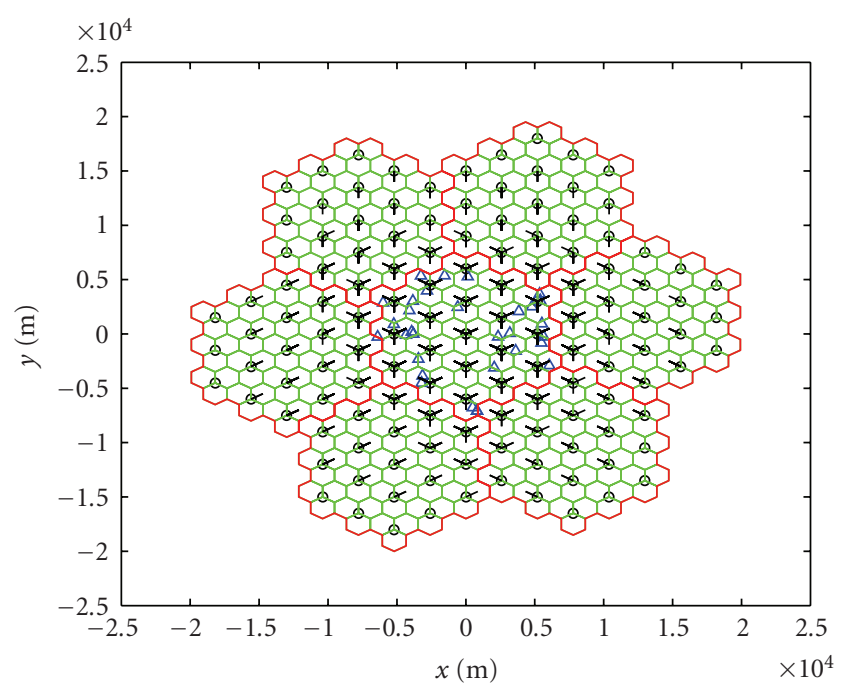

$\circ$ BS

$\triangle$ MS

- Beam direction

FIGURE 1: The considered network layout in the simulation.

TABle 1: Simulation parameters.

\begin{tabular}{ll}
\hline Parameters & Value \\
\hline Layout & $\begin{array}{l}19 \text { cells, } 3 \text { sectors/cell-wrap } \\
\text { around }\end{array}$ \\
Propagation scenario & Base coverage urban \\
Cell radius & $1000 \mathrm{~m}$ \\
Maximum MS transmit power & $24 \mathrm{dBm}$ \\
Maximum antenna gain & $17 \mathrm{dBi}$ \\
Scheduling interval & 10 transmission time interval \\
1 transmission time interval & $1 \mathrm{~ms}$ \\
Thermal noise density & $-174 \mathrm{dBm} / \mathrm{Hz}$ \\
Number of users & 30 in 19 cells \\
BS receiver antenna array & ULA \\
BS receiver antenna elements & 2 \\
UE antenna & 1 \\
Number of BSs for coordinate & $1,2,3$ \\
reception & $0,8 \mathrm{~dB}$ \\
SINR constraint per MS & $3 \mathrm{~km} / \mathrm{h}$ \\
MS speed & Log-Normal, $8 \mathrm{~dB}$ standard \\
Shadow fading & deviation \\
Shadowing correlation & independent \\
Down tilt angle & $8 \mathrm{degree}$ \\
\hline
\end{tabular}

As shown in Figure 1, the central 57 sectors are the original sectors while the outer sectors are the copies of the central sectors. The edge effect is then eliminated by wrapping around the network [25]. Other simulation parameters are listed in Table 1. For convenience, we assume that $\left|\pi_{1}\right|=$ $\left|\pi_{2}\right|=\cdots=\left|\pi_{K}\right|$ in all simulations. The SINR constraints are set to $0 \mathrm{~dB}$ and $8 \mathrm{~dB}$ in order to simulate voice and data oriented systems.

The large-scale fading parameters, including path loss, shadow fading and antenna beam pattern gain are from International Telecommunication Union (ITU) [26]. For simplicity, we drop the BS and link index. The general sector antenna field pattern can be modeled as

$$
A_{A}(\theta)=-\min \left[12\left(\frac{\theta}{\theta_{3 \mathrm{~dB}}}\right), A_{m}\right], \quad-180^{\circ} \leq \theta \leq 180^{\circ} \text {, }
$$

where $\theta$ is the arrival angle, $\theta_{3 \mathrm{~dB}}$ is the $3 \mathrm{~dB}$ beamwidth which is $70^{\circ}$ for 3 -sector cell and $A_{m}=20 \mathrm{~dB}$ is the maximum attenuation. The general path loss model is given by

$$
\begin{aligned}
P_{\text {pathloss }}= & \left(44.9-6.55 \log 10\left(h_{\mathrm{BS}}\right)\right) \log 10\left(d_{\mathrm{BS}-\mathrm{MS}}\right) \\
& +34.46+5.83 \log 10\left(h_{\mathrm{BS}}\right)+C_{\text {pathloss }} * \log 10\left(\frac{f_{c}}{5}\right),
\end{aligned}
$$

where $h_{\mathrm{BS}}$ is the BS antenna hight, $d_{\mathrm{BS}-\mathrm{MS}}$ is the distance between considered BS and MS, $C_{\text {pathloss }}$ is the frequency dependence factor, which is 23 in urban macro-non-line of site scenario and $f_{c}$ is the carrier frequency. Finally, the shadow fading is log normal distributed with standard deviation of $8(\mathrm{~dB})$. The MS antenna is assumed to be placed at $1.5 \mathrm{~m}$ above the ground [26].

4.1. Performance Analysis of the Proposed Algorithm. First, the feasibilities against the number of MSs of the alternative schemes are compared in Figure 2, where the SINR constraint per MS is $0 \mathrm{~dB}$. As an important benchmark, we also give the performance of the full cooperative scheme, where all BSs jointly process their received signals as a huge virtual MIMO system. This gives us the theoretical upper bound of the system performance, which is shown by the black solid line in the figure. Obviously, Algorithm 1 with $\left|\pi_{k}\right|=$ 3 can support more MSs than other partially cooperative schemes as it achieves the highest feasibility. Compared with the fully cooperative network MIMO, it still has a perceptible gap when the number of MSs increases up to 100 or higher. From the complexity viewpoint, Algorithm 2 is the simplest scheme. However, it always has the worst performance amongst all compared schemes with the same number of $\left|\pi_{k}\right|$. When $\left|\pi_{k}\right|$ is increased, the performance gap reduces. By setting $R_{1}=R_{2}=\cdots=R_{K}=5$, we obtain the performances of Algorithm 3 with different $\left|\pi_{k}\right|$ values. Among all simplified schemes, we notice that Algorithm 3 always achieve higher feasibilities than others with the same $\left|\pi_{k}\right|$. Another interesting result could be found that Algorithm 3 shows a little better performance than Algorithm 4, where $R_{k}$ is dynamically changed based on the measured RSS values of MSs. The reason is that the threshold $P_{L}$ is not large, thus the number of BSs involved in the joint receiving is less than 5 as in Algorithm 3. By setting $P_{L}$ to a larger value, a better performance from Algorithm 4 can be expected, besides a higher complexity. A similar behavior 


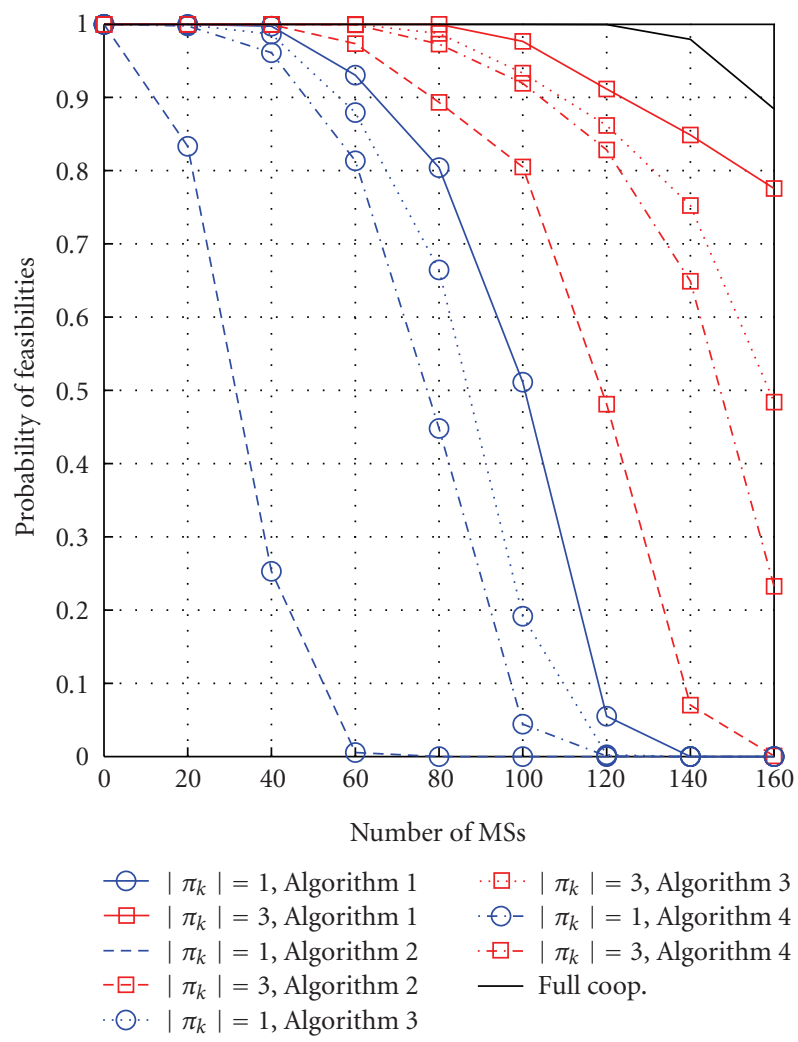

FIgURe 2: Comparison of probabilities of feasibility versus the number of MSs with the SINR constraint of $0 \mathrm{~dB}$.

could be also found in Figure 3 where the SINR constraint per MS is set as $8 \mathrm{~dB}$.

In Figure 4, the cumulated distribution functions (CDF) of transmit power per MS of alternative schemes are plotted. In this comparison, $30 \mathrm{MSs}$ are uniformly distributed over the whole interested area and the SINR constraint is set also as $0 \mathrm{~dB}$ firstly. In this comparison, only the powers obtained under the feasible channel realizations are counted. As we expected, Algorithm 1 with $\left|\pi_{k}\right|=3$ achieves a very close performance to the upper bound in this comparison, where the difference is less than $0.8 \mathrm{~dB}$ at most. Naturally, its performance is getting worse when $\left|\pi_{k}\right|$ is reduced to 1 . Same as the feasibility comparison, Algorithm 2 still performs the worst with the same $\left|\pi_{k}\right|$ while Algorithm 3 shows very good performance. Compared with Algorithm 2, the performance gap between Algorithms 3 and 1 is almost negligible. Moreover, Algorithm 3 shows slightly better performance than Algorithm 4 like in the previous comparison. The similar findings can be found also in Figure 5, where the SINR constraint is $8 \mathrm{~dB}$ per MS.

4.2. Comparison of the Proposed Algorithm with Inter-IntraSite CoMP. The inter-site and intra-site CoMPs, are in fact two special cases of full set selection, that is, Algorithm 1, with $R_{k}=3$ and $\left|\pi_{k}\right|=3$. In the inter-site and intrasite CoMP, BSs have been grouped based on the geographic information. The receive strategies of the two schemes are also chosen from a single-element set. The cell combination

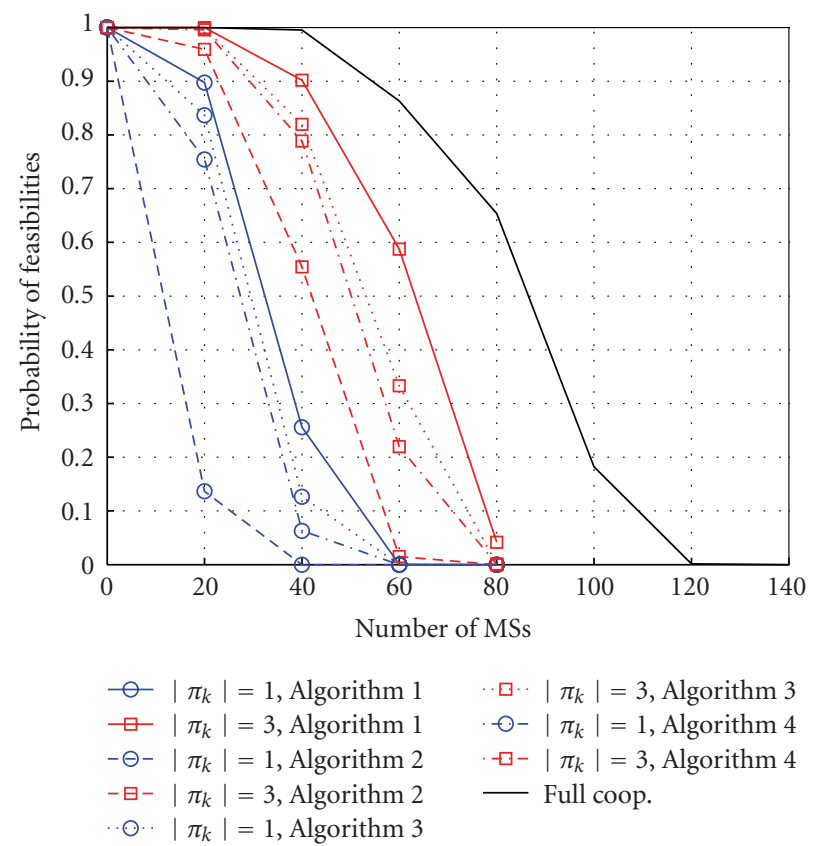

FIGURE 3: Comparison of probabilities of feasibility versus the number of MSs with the SINR constraint of $8 \mathrm{~dB}$.

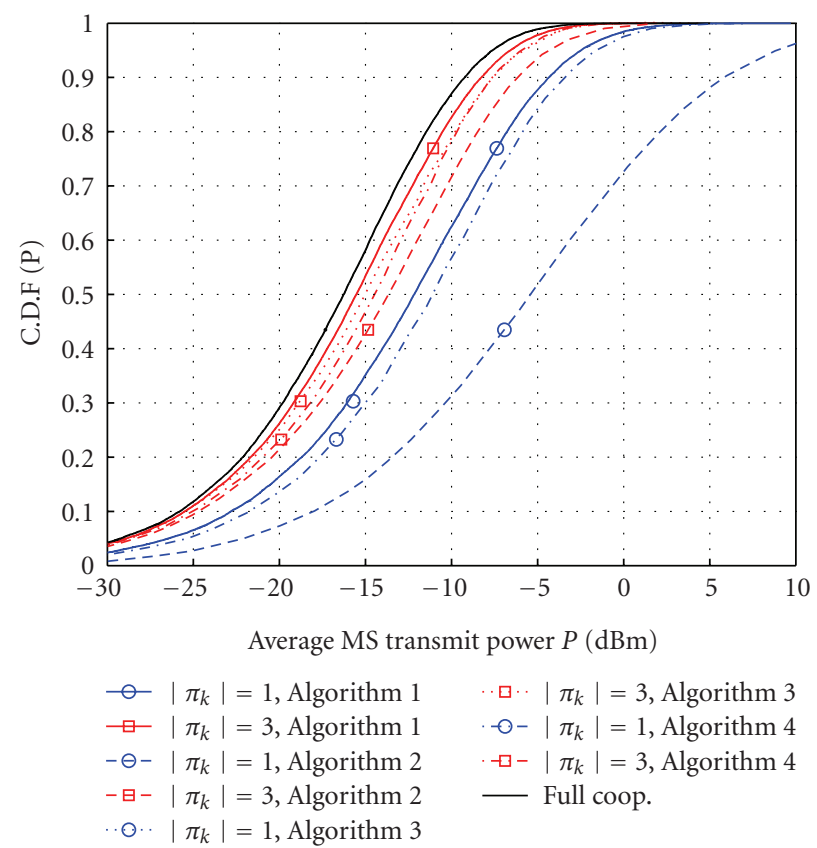

FIGURE 4: Comparison of CDFs of transmit power of different sizes of joint processing MBS with the SINR constraint of $0 \mathrm{~dB}$.

set of the CoMP is fixed, while the single-element set has an adaptive combination. With minor modifications, our proposed TPC algorithm can be easily applied to inter-site and intra-site CoMP. An illustration of the inter-intra-site cooperation is included in Figure 6.

The feasibility comparisons are shown first in Figures 7 and 8 , where the SINR constraint per MS is $0 \mathrm{~dB}$ and $8 \mathrm{~dB}$, 


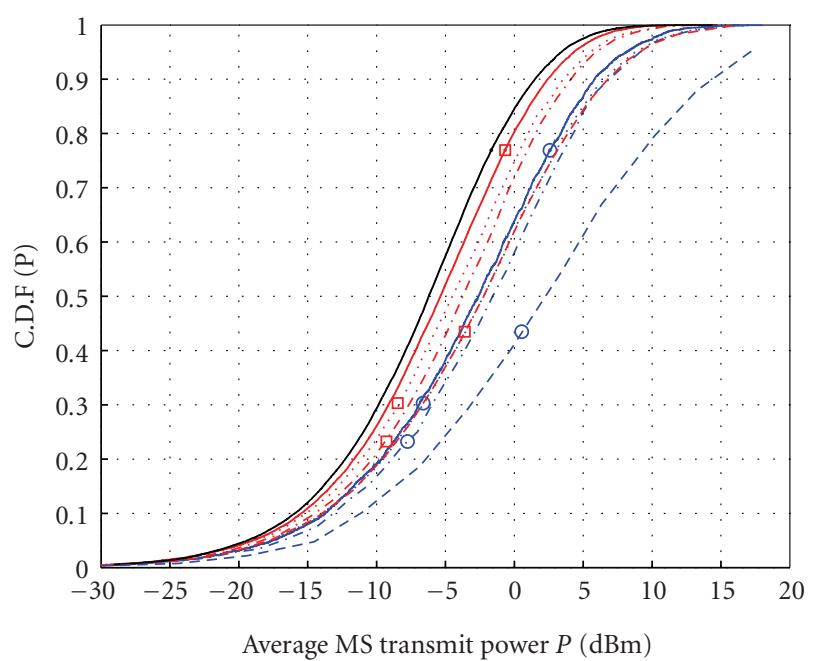

$$
\begin{aligned}
& -\circ\left|\pi_{k}\right|=1 \text {, Algorithm } 1 \quad \cdots \square \cdot \cdot\left|\pi_{k}\right|=3 \text {, Algorithm } 3 \\
& \square-\left|\pi_{k}\right|=3 \text {, Algorithm } 1 \quad--\left|\pi_{k}\right|=1 \text {, Algorithm } 4 \\
& -\ominus-\left|\pi_{k}\right|=1 \text {, Algorithm } 2 \quad \bullet-\left|\pi_{k}\right|=3 \text {, Algorithm } 4 \\
& - \text { - }\left|\pi_{k}\right|=3 \text {, Algorithm } 2 \text { Full coop. } \\
& \text {.๑. }\left|\pi_{k}\right|=1 \text {, Algorithm } 3
\end{aligned}
$$

FIGURE 5: Comparison of CDFs of transmit power of different sizes of joint processing MBS with the SINR constraint of $8 \mathrm{~dB}$.

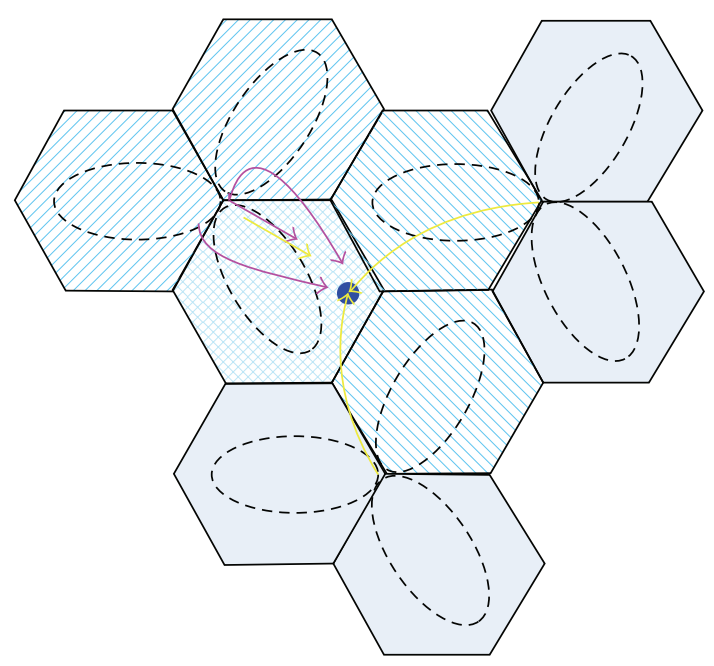

\footnotetext{
$>$ Intercell coop.

$\longrightarrow$ Intracell coop.

- MS
}

Figure 6: An illustration of inter-site and intrasite reception schemes.

respectively. The adaptive scheme shows obvious advantage over the conventional schemes in both two figures. It is also observed that the inter-site cooperation achieves higher probability of feasibility than the intra-site cooperation.

The CDF curves of the average transmit power are plotted in Figures 9 and 10 with the same SINR constraint settings as the feasibility comparisons. When the transmit power is small, we see that the intra-site cooperation

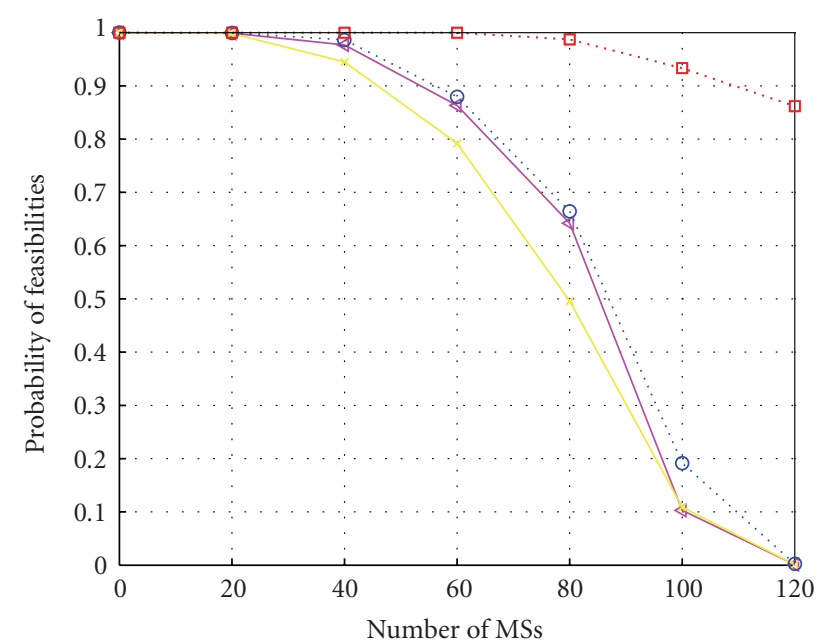

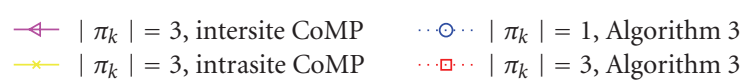

Figure 7: Comparison of probabilities of feasibility of intra-site, inter-site cooperation and proposed adaptive reception schemes. Again SINR constraint is $0 \mathrm{~dB}$.

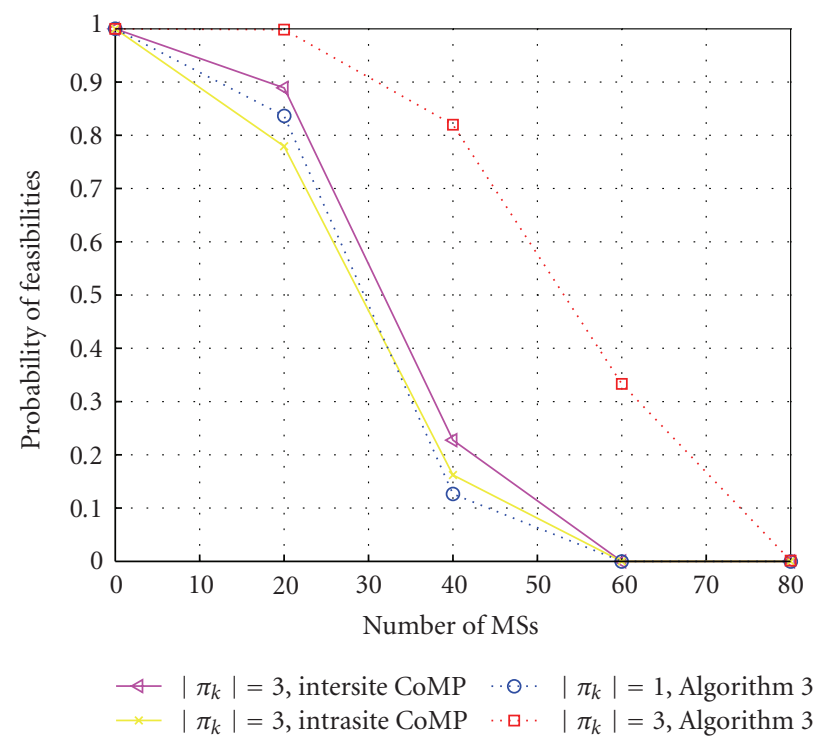

FIgURE 8: Comparison of probabilities of feasibility of intra-site, inter-site cooperation and proposed adaptive reception schemes. Again SINR constraint is $8 \mathrm{~dB}$.

performs a little better than the inter-site cooperation. As the required transmit power is increased, the inter-site cooperation starts to outperform the intra-site cooperation. The reason is that the small and large transmit powers usually indicates the cases that the MS is located at the cell center and cell edge, respectively. The sector-beam attenuation, rather than the path loss, dominates the channel gains in the cell-center case, while in the cell-edge case, the path loss becomes more significant. From the CDF results, the obvious advantage of the adaptive scheme is still observed. Algorithm 3 with $\left|\pi_{k}\right|=1$ sometimes outperforms 3-sector 


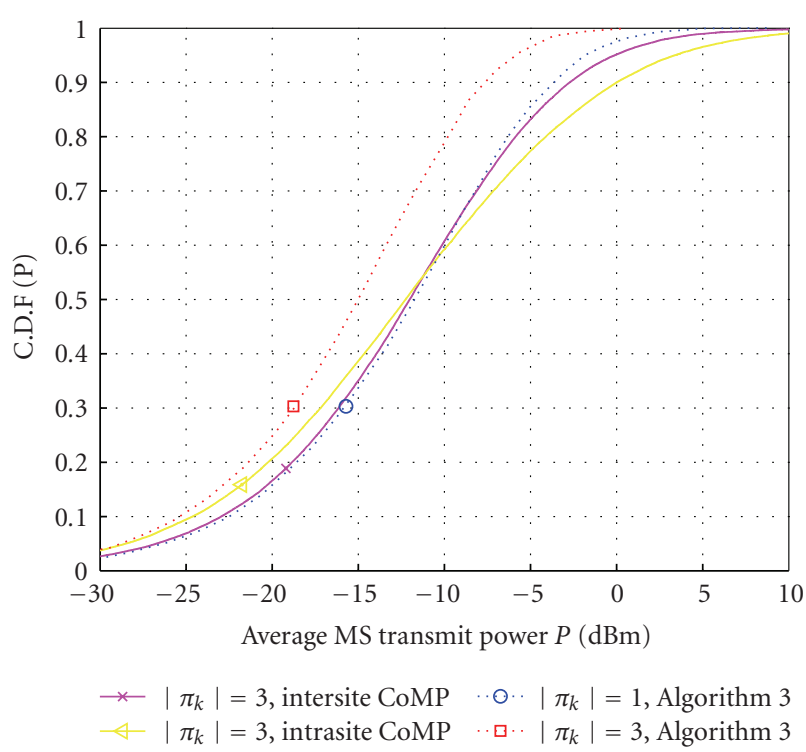

FIgure 9: Comparison of CDFs of transmit power of inter-site, intra-site and adaptive reception schemes with the SINR constraint of $0 \mathrm{~dB}$.

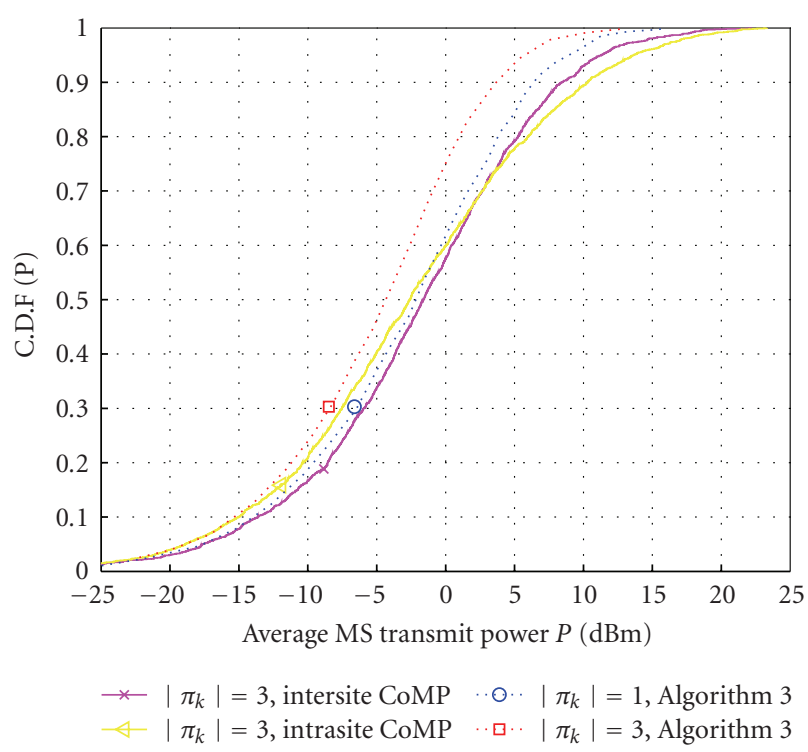

FIgURE 10: Comparison of CDFs of transmit power of inter-site, intra-site and adaptive reception schemes with the SINR constraint of $8 \mathrm{~dB}$.

CoMP is because Algorithm 3 is near optimal and the best sector is chosen based on small scale fading out of 5 candidate sectors, while the fixed-cluster CoMP is only chosen based on large-scale fading parameters.

4.3. Complexity Analysis. The complexity of Algorithm 1 is composed of two parts including the convergence speed, that is, the number of iterations it takes to converge, and the number of candidates in the exhaustive search set at each iteration. For convenience, we do not consider the

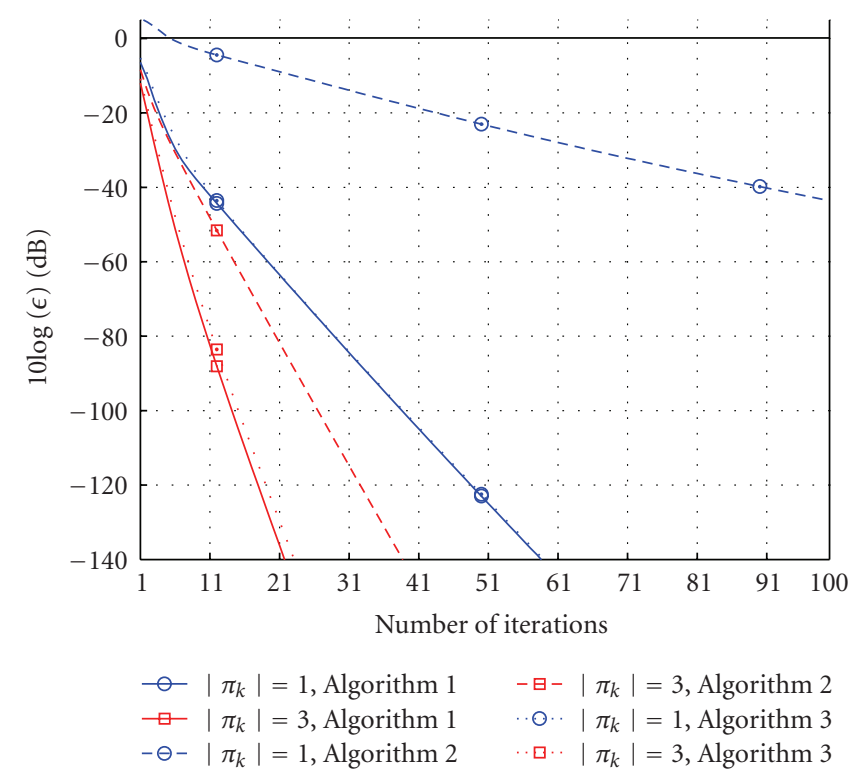

Figure 11: Comparison of convergence speed of different algorithms.

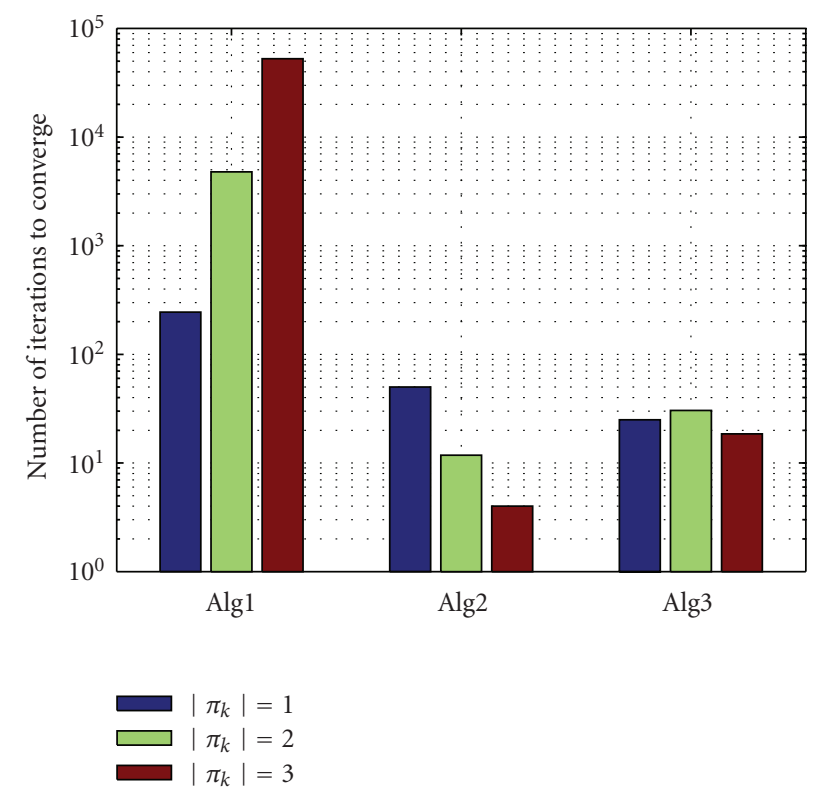

Figure 12: Comparison of total number of iterations for different algorithms and $\pi_{k}$.

complexity of Algorithm 4 in this comparison, as it does not show better performance than Algorithm 3 and its complexity of the second part can not be directly calculated.

In order to see the convergence behavior of the alternative schemes, we first fix the maximum number of the iterations to be 100 and skip step (3) in Algorithm 1. At each iteration $t$, we calculate the average transmit power per MS, that is, $\overline{\mathbf{p}}^{[t]}=P_{\mathrm{tot}}^{[t]} / K$. Later, the difference between two consecutive iterations is computed as $\epsilon=\bar{p}^{[t]}-\bar{p}^{[t+1]}$. After averaging over 1000 channel realizations, we illustrate $\epsilon$ in Figure 11 . 
As the iteration index $t$ is increased, Algorithm 1 shows the fastest convergence speed with the same value of $\left|\pi_{k}\right|$. For example, when setting $\left|\pi_{k}\right|=3$ and $\epsilon=5 \mathrm{e}-3$, Algorithm 1 takes about 1.8 iterations to converge, while Algorithms 2 and 3 take 4 and 1.85 iterations, respectively. In addition, the larger the $\left|\pi_{k}\right|$ values, the faster convergence speed for all schemes.

Then by setting $B=57, R_{k}=5$ and $\epsilon=5 \mathrm{e}-3$, we can calculate the product of the number of iterations and the number of the candidates in the set to be exhaustively searched, which, to some extent, represents the total complexity of the algorithm. The obtained results of the alternative schemes with different values of $\left|\pi_{k}\right|$ are shown in Figure 12. Although Algorithm 1 shows the fastest convergence speed in the previous comparison, it still has significantly higher complexity than Algorithms 2 and 3 do. When $\left|\pi_{k}\right|=1$, Algorithms 2 and 3 have about the same complexities. But when $\left|\pi_{k}\right|$ is increased to 2 and 3, the complexity of Algorithm 2 is reduced significantly while that of Algorithm 3 does not change so much. As the total complexity depends on both convergence speed and number exhaustive search, thus, Algorithm 3 almost has the similar complexity for different $\left|\pi_{k}\right|$.

\section{Conclusion}

The joint TPC and receive beamforming with adaptive MBS selection for uplink communications are studied. To minimize the total transmit power, an algorithm that optimally solves the problem is presented accordingly. As the optimal MBS selection involves the exhaustive search per MS over all BSs, several simplified schemes with different BS preselection schemes are presented. The proposed algorithm with both optimal and simplified MBS selection is evaluated by the system-level simulations. The results show that, using the simplified scheme, better tradeoffs between the complexity and the performance can be achieved. We also compare the proposed adaptive scheme to the conventional fixed inter-cell and intra-site CoMP, where the obvious advantages of the adaptive scheme are observed.

\section{References}

[1] S. A. Gandhi, R. Vijayan, D. J. Goodman, and J. Zander, "Centralized power control in cellular radio systems," IEEE Transactions on Vehicular Technology, vol. 42, no. 4, pp. 466468, 1993.

[2] S. A. Grandhi, R. Vijayan, and D. J. Goodman, "Distributed power control in cellular radio systems," IEEE Transactions on Communications, vol. 42, no. 2, pp. 226-228, 1994.

[3] G. J. Foschini and Z. Miljanic, "Simple distributed autonomous power control algorithm and its convergence," IEEE Transactions on Vehicular Technology, vol. 42, no. 4, pp. 641-646, 1993.

[4] R. D. Yates and C. Y. Huang, "Integrated power control and base station assignment," IEEE Transactions on Vehicular Technology, vol. 44, no. 3, pp. 638-644, 1995.

[5] R. D. Yates, "Framework for uplink power control in cellular radio systems," IEEE Journal on Selected Areas in Communications, vol. 13, no. 7, pp. 1341-1347, 1995.
[6] F. Rashid-Farrokhi, L. Tassiulas, and K. Liu, "Joint optimal power control and beamforming in wireless networks using antenna arrays," IEEE Transactions on Communications, vol. 46, no. 10, pp. 1313-1324, 1998.

[7] F. Rashid-Farrokhi, K. J. R. Liu, and L. Tassiulas, "Transmit beamforming and power control for cellular wireless systems," IEEE Journal on Selected Areas in Communications, vol. 16, no. 8, pp. 1437-1449, 1998.

[8] M. Schubert and H. Boche, "Solution of the multiuser downlink beamforming problem with individual SINR constraints," IEEE Transactions on Vehicular Technology, vol. 53, no. 1, pp. 18-28, 2004.

[9] M. Bengtsson and B. Ottersten, "Optimal downlink beamforming using semidefinite optimization," in Proceedings of the Annual Allerton Conference on Communications, Control and Computing, pp. 987-996, September 1999.

[10] S. Papavassiliou and L. Tassiulas, "Joint optimal channel base station and power assignment for wireless access," IEEE/ACM Transactions on Networking, vol. 4, no. 6, pp. 857-872, 1996.

[11] F. Rashid-Farrokhi, K. J. R. Liu, and L. Tassiulas, "Downlink power control and base station assignment," IEEE Communications Letters, vol. 1, no. 4, pp. 102-104, 1997.

[12] J. Yang and D. K. Kim, "Multi-cell uplink-downlink beamforming throughput duality based on Lagrangian duality with per-base station power constraints," IEEE Communications Letters, vol. 12, no. 4, pp. 277-279, 2008.

[13] S. Venkatesan, A. Lozano, and R. Valenzuela, "Network MIMO: overcoming intercell interference in indoor wireless systems," in Proceedings of the 41st Asilomar Conference on Signals, Systems and Computers (ACSSC '07), pp. 83-87, November 2007.

[14] W. Li, M. Juntti, X. Lu, A. Tolli, E. Kunnari, and O. Piirainen, "Adaptive joint multi-cell reception with uplink power control and beamforming(invited)," in Proceedings of the 5th International ICST Conference on Communications and Networking in China (CHINACOM '10), China, August 2010.

[15] M. K. Karakayali, G. J. Foschini, and R. A. Valenzuela, "Network coordination for spectrally efficient communications in cellular systems," IEEE Wireless Communications, vol. 13, no. 4, Article ID 1678166, pp. 56-61, 2006.

[16] S. Shamai, O. Somekh, O. Simeone et al., "Cooperative multicell networks: impact of limited-capacity backhaul and interuser links," in Proceedings of the International Symposium on Information Theory, Durnstein, Austria, October 2007.

[17] A. Sanderovich, O. Somekh, H. V. Poor, and S. Shamai, "Uplink macro diversity of limited backhaul cellular network," IEEE Transactions on Information Theory, vol. 55, no. 8, pp. 3457-3478, 2009.

[18] O. Simeone, O. Somekh, H. V. Poor, and S. Shamai (Shitz), "Downlink multicell processing with limited-backhaul capacity," EURASIP Journal on Advances in Signal Processing, vol. 2009, Article ID 840814, 10 pages, 2009.

[19] S. A. Ramprashad and G. Caire, "Cellular vs. network MIMO: a comparison including the channel state information overhead," in Proceedings of the 20th IEEE International Symposium on Personal, Indoor and Mobile Radio Communications, PIMRC, Tokyo, Japan, September 2009.

[20] S. A. Ramprashad, G. Caire, and H. C. Papadopoulos, "Cellular and network MIMO architectures: MU-MIMO spectral efficiency and costs of channel state information," in Proceedings of the Asilomar Conference on Signals, Systems and Computers, pp. 1811-1818, Pacific Grove, Calif, USA, November 2009. 
[21] G. Caire, S. A. Ramprashad, and H. C. Papadopoulos, "Rethinking network MIMO: cost of CSIT, performance analysis, and architecture comparisons," in Proceedings of the Information Theory and Applications Workshop (ITA '10), pp. 386-395, San Diego, Calif, USA, February 2010.

[22] H. Zhang, N. B. Mehta, A. F. Molish et al., "Asychronous interference miigation in cooperative base station system," IEEE Transactions on Wireless Communications, vol. 7, no. 1, pp. 155-165, 2008.

[23] H. Zhang, N. B. Mehta, A. F. Molisch, J. Zhang, and H. Dai, "On the fundamentally asynchronous nature of interference in cooperative base station systems," in Proceedings of the IEEE International Conference on Communications (ICC '07), pp. 6073-6078, June 2007.

[24] M. Chiang, P. Hande, T. Lan, and C. W. Tan, "Power control in wireless cellular networks," Foundations and Trends in Networking, vol. 2, no. 4, pp. 381-533, 2007.

[25] T. Hytonen, "Optimal wrap-around network simulation," Research Reports, Helsinki University of Technology, Helsinki University of Technology Institute of Mathematics, Espoo, Finland, 2001.

[26] International Telecommunication Union, "Guidelines for evaluation of radio interface technologies for IMT-Advanced," November 2008, http://www.itu.int/pub/R-REP-M.2135-2008 len. 